\title{
The test of time
}

\author{
Elizabeth Loder head of research
}

The BMJ

Which of today's headline breakthroughs will still be judged important years from now? Many treatments don't stand the test of time: they are effective in the short run but fail to produce lasting health benefits. It can be difficult to evaluate the long term effects of medical interventions, especially when outcomes of interest may not occur for decades. Such is the case with antibiotic treatment for Helicobacter pylori infection. For this reason, research reported this week by Jing-Yu Zhang and colleagues deserves our attention (doi:10.1136/bmj.15016). The authors report on over two decades of follow-up of a randomised, placebo controlled trial of brief antibiotic treatment for $H$ pylori (in trial participants who tested positive for it) and longer term vitamin and garlic supplementation. Treatment of $H$ pylori infection and vitamin supplementation were both associated with meaningful reductions in the development of gastric cancer, and all three interventions were associated with a reduced risk of death related to cancer. The study took place in China, where the risk of gastric cancer is high and vitamin deficiencies are common. It remains uncertain whether the results can be generalised to better nourished populations or those with a lower baseline risk of gastric cancer.

What about the long term success of interventions to lower the risk of type 2 diabetes? Dianna Magliano and colleagues are optimistic that preventive strategies, such as reducing consumption of sugar sweetened beverages, may be paying off (doi:10.1136/bmj.15003). They conclude that incidence of type 2 diabetes seems to have plateaued or even fallen in some parts of the developed world. That would be welcome news indeed, but editorialists Mike Lean and colleagues caution against premature claims of victory (doi:10.1136/bmj.15407). The prevalence of obesity continues to rise in most of the world, especially developing countries, and weight is the most important risk factor for diabetes. Weight management strategies, they say, are the way forward if we want to intervene effectively.

Another unsolved problem is how to empower healthcare workers to report concerns about substandard care. It's not difficult to understand why frontline staff, who are best placed to identify dangerous practices, are reluctant to come forward. They see everything, and have the most direct experience to offer, but institutions and those in power have never been kind to whistle blowers. Russell Mannion and Huw Davies point out that improved reporting isn't enough (doi:10.1136/bmj.14944): "It is widely recognised that speaking up takes courage, but it is less obvious that it may also require courage to accept that the concerns raised have merit." Effective strategies also need to protect "those who relay unwelcome messages" in addition to those who spoke up in the first place. Promising interventions exist, they say, but more time, and further development and testing, are needed to evaluate their impact. 